\title{
Load Balancing and Continuous Quadratic Programming
}

\author{
William W. Hager \\ Department of Mathematics, University of Florida, \\ 358 Little Hall, Gainesville, FL 32611-8105. hager@math.ufl.edu
}

\begin{abstract}
A quadratic programming approach is described for solving the graph partitioning problem, in which the optimal solution to a contin uous problem is the exact solution to the discrete graph partitioning problem. We discuss techniques for appro ximatingthe solution to the quadratic program using gradient projections, preconditioned conjugate gradien ts, and a blok exchange method.
\end{abstract}

\section{Extended Abstract}

In a parallel computing environment, tasks can be modelled as nodes on a graph and the communication links betw een tasks as edges on the graph. The problem of assigning the tasks to different processors while minimizing the communication betw een processors is equilent to partitioning the nodes of the graph into sets chosen so that the number of edges connecting nodes in different sets is as small as possible. The graph partitioning problem was first exposed by Kernighan and Lin in the seminal paper in 1970. Since Sahni and Gonzales show in 1976 that graph partitioning is an NP hard problem, exact solutions can be computed only when the number of nodes is small. To solv e large problems, approimation techniques have been developed that include exchange techniques, spectral methods, geometric methods, and multilev el methods. Our approad to the graph partitioning problem is based a quadratic programming formulation. For the problem of partitioning an $n$ node graph into $k$ sets, w e exhibit a quadratic programming problem (quadratic cost function and linear equality and inequality constraints) in $n k$ variables $x_{i j}, 1 \leq i \leq n, 1 \leq j \leq k$, where $x_{i j}$ is a contin uous variable taking values on the interval $[0,1]$. This quadratic program is equivalent to the discrete graph partitioning problem in the sense that there exists an optimal $0 / 1$ solution to the quadratic program and the assignment of node $i$ to set $j$ if $x_{i j}=1$ is optimal in the graph partitioning problem. Based on this equivalence betw een graph partitioning and a quadratic problem, we have applied tools from optimization theory to solve the graph partitioning problem. In this talk, we discuss tec hniques for approimating the solution to the quadratic program using gradient projections, preconditioned conjugate gradients, and a block exc hange method. The advantages and disadvantages of the optimization approach compared to other approaches to graph partitioning are also examined. For papers related to this talk, see http://www.math.ufl.e du Thager. 